\title{
New developments of the CARTE thermochemical code: II - Evaluation of the accuracy of the KLRR perturbation theory and treatment of mixtures
}

\author{
N. Desbiens ${ }^{\mathrm{a}}$ and V. Dubois
}

CEA, DAM, DIF, 91297 Arpajon, France

\begin{abstract}
The KLRR perturbation theory has been initially proposed by Kang et al. in order to compute the equation of state (EOS) of a fluid of soft spheres represented by an EXP-6 potential. Victorov and Gubin have recently improved this method and they showed that, compared to other theories (namely HMSA/C and MCRSR), this improved KLRR theory turns out to be more accurate. In the first part of this paper, the comparison is extended to a larger range of temperature and stiffness of potential. Then, in order to use such a perturbation theory in a thermochemical code, the treatment of mixtures has to be handled since the KLRR method is expressed for a pure fluid. Usually, the Van-der-Waals one fluid mixing rule (VdW-1f) is employed. It consists in averaging the mixture by an effective fluid. We also intend here to re-evaluate this well-known and largely used model.
\end{abstract}

\section{Introduction}

In the fiel of detonation science, shock physics or geophysics, there is a need for accurate high pressure equations of state for fluids (pure or mixtures). To this end, different potential models and computational methods have been evaluated. Concerning the potential models, it has quickly appeared that the exponential-6 potential (EXP-6) is particularly well suited for high pressure regimes thanks to its stiffness at short distance. For computational methods, different theories have been proposed and give accurate results when compared to reference Monte Carlo simulation data. Perturbation theories such as the well-known MCRSR [1] or the original KLRR [2] have been developed and compare well with Monte Carlo simulation data. In our CARTE thermochemical code [3], the method used is MCRSR [4]. Integral theories, particularly the HMSA method [5] and the HMSA/MC method [6], can also provide accurate results. Especially, Fried and Howard [6] obtained very accurate results by adding a correcting term to the HMSA integral equation in order to fit the Monte Carlo simulation data. Recently, Victorov and Gubin [7] have improved the KLRR method. They showed that the accuracy of their new EOS exceeds that of the MCRSR and HMSA/MC ones when compared to Monte Carlo simulation data of Fried and Howard [6]. It has to be noted that the improved KLRR theory does not use any correcting or empirical term. The reference Monte Carlo simulation data provided by Fried and Howard are restricted to $T^{*}=\{5-20-100\}$ for the reduced temperature and $\alpha=\{11.5-13.5-15.5\}$ for the stiffness of the potential. We intend here to extend this reference Monte Carlo simulation database to check the accuracy of the MCRSR and the improved KLRR theories. The aim is also to create a reference database that can be used to quantify the accuracy of any new perturbation theory or any new integral equation or even as a part of a fully Monte Carlo EOS (expressed by means of Chebyshev coefficients).

To compute the properties of a mixture by means of perturbation theory, the parameters of the potential of the effective fluid (indiced $x$ ) have to be determined. By conformal solution theory [8] or

\footnotetext{
a e-mail: nicolas.desbiens@cea.fr
} 


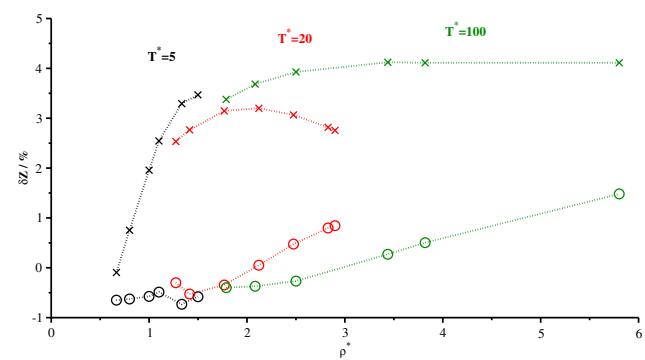

Fig. 1. Relative differences $\delta Z$ along the three isotherms as a function of the reduced density $\rho^{*}$ for $\alpha=11.5$. Crosses correspond to MCRSR calculations and circles correspond to improved KLRR calculations.

by virial expansion [9], one can find relatively simple mixing rules for hard sphere systems (Eq. (1)) or Lennard-Jones 6-12 systems (Eqs. (1) and (2)):

$$
\begin{aligned}
\sigma_{x}^{3} & =\sum_{i j} x_{i} x_{j} \sigma_{i j}^{3} \\
\epsilon_{x} \sigma_{x}^{3} & =\sum_{i j} x_{i} x_{j} \epsilon_{i j} \sigma_{i j}^{3}
\end{aligned}
$$

where $x_{i}$ is the fraction of specie $i$ and $\sigma_{i j}$ is the interaction parameter between specie $i$ and specie $j$. For such rules, it can be showed that at the zero order term the Helmholtz free energy of the effective fluid is equal to the Helmholtz free energy of the mixture. In the case of EXP-6 potentials, analytical expressions derived from virial expansion are not tractable [10]. Ree [11] proposed a mixing rule for $\alpha$ based on an extension of rules 1 and 2:

$$
\alpha_{x} \epsilon_{x} \sigma_{x}^{3}=\sum_{i j} x_{i} x_{j} \alpha_{i j} \epsilon_{i j} \sigma_{i j}^{3}
$$

The accuracy of rules 1, 2 and 3 (hereafter noted VdW-1f) has been checked by comparing total pressure and total energy of mixtures with respect to Monte Carlo simulation data of $\mathrm{H}_{2}-\mathrm{He}$ binary mixtures. To our opinion, such a comparison can give misleading results since the excess pressure for example corresponds to a small part of the total pressure. Nonetheless, these mixing rules have been largely used but, to our knowledge, their accuracy has never been precisely quantified on excess properties. In the second part of this paper, we evaluate the precision of the VdW-1f rules. We also perform an optimization of some coefficients of the mixing rules in order to reproduce Monte Carlo simulation data of excess properties.

\section{Comparison of improved KLRR and MCRSR theories}

\subsection{Comparison to Fried and Howard reference database}

Fried and Howard computed the compressibility factor $Z$ and the reduced energy $U^{*}$ along three isotherms $\left(T^{*}=5-20-100\right)$ for three values of $\alpha(11.5-13.5-15.5)$ by Monte Carlo simulations. These simulation data are the statistically exact results for EXP-6 potentials. Victorov and Gubin [7] have compared the improved KLRR results to these reference data. We also computed these quantities with the MCRSR perturbation theory. Relative differences $\delta Z$ and $\delta U$ enable to quantify the gap with regard to Monte Carlo data (MC):

$$
\delta Z=\frac{Z-Z_{M C}}{Z_{M C}} \quad \delta U=\frac{U^{*}-U_{M C}^{*}}{\left|U_{M C}^{*}\right|}
$$

We report in Fig. 1 the relative difference $\delta Z$ for $\alpha=11.5$. This quantity lies between $-1 \%$ and $1 \%$ for KLRR and between $2 \%$ and $4 \%$ for MCRSR. Fried and Howard computed averaged relative 
Table 1. Averaged relative differences for HMSA/C, HMSA/MC [6], MCRSR and improved KLRR theories.

\begin{tabular}{ccc}
\hline & $\delta Z(\%)$ & $\delta U(\%)$ \\
\hline MCRSR & 2.08 & 2.44 \\
KLRR & 0.59 & 0.83 \\
HMSA/C & 0.69 & 1.65 \\
HMSA/MC & 0.19 & 0.28 \\
\hline
\end{tabular}

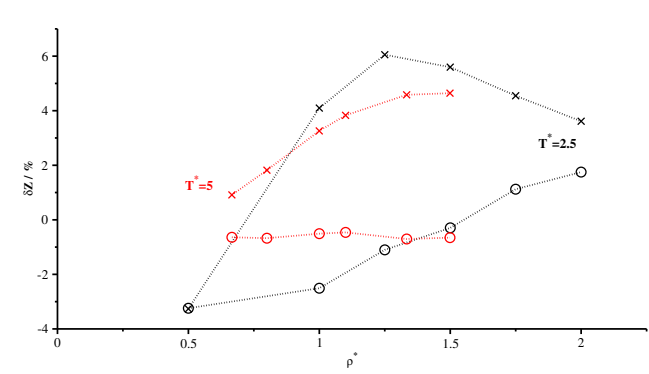

Fig. 2. Relative differences $\delta Z$ along two isotherms as a function of the reduced density $\rho^{*}$ for $\alpha=10.5$. Crosses correspond to MCRSR calculations and circles correspond to improved KLRR calculations.

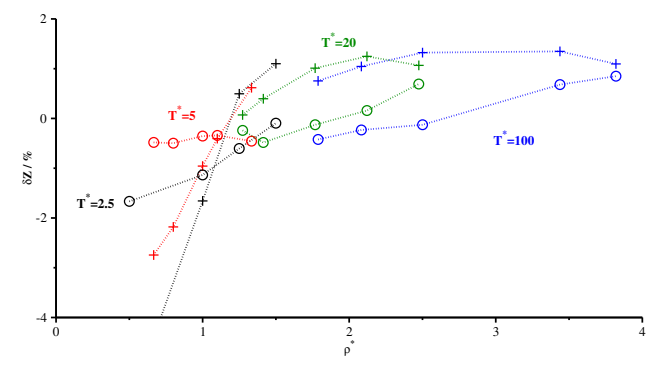

Fig. 3. Relative differences $\delta Z$ along four isotherms as a function of the reduced density $\rho^{*}$ for $\alpha=17.5$. Crosses correspond to MCRSR calculations and circles correspond to improved KLRR calculations.

differences of $Z$ and $U^{*}$ for the HMSA/C and HMSA/MC methods. Their results are reported in Table 1 together with the results concerning KLRR and MCRSR. Improved KLRR appears to be very accurate compared to MCRSR and HMSA/C theories. HMSA/MC method is a little bit more accurate but this method contains an empirical term fitted on the Monte Carlo results. This point reduces the appeal of the HMSA/MC method. In the end, the improved KLRR theory has been implemented in our CARTE thermochemical code dedicated to calculation of the properties of detonation products mixtures.

\subsection{Extension of the database}

The values of $\alpha$ used by Fried and Howard are quite common. But some fluids can have lower values of $\alpha$ (soft potentials) or higher values (hard potentials). Moreover as we hope to introduce experimental results provided by Diamond Anvil Cell experiments in the calibration of the parameters of the potentials [4], we want to quantify the accuracy of improved KLRR in the low temperature regime $\left(T^{*}=2.5\right)$. To this end, Monte Carlo simulations have been performed with the GIBBS simulation code $^{1}$ for $T^{*}=2.5$, for $\alpha=10.5$ and for $\alpha=17.5$ in the same way as described in [6]. Monte Carlo simulation data, MCRSR and KLRR calculations together with technical details will be detailed in a paper to come [12]. The results concerning $\alpha=10.5$ and $\alpha=17.5$ are summarized in figures 2 and 3 .

\footnotetext{
${ }^{1}$ The GIBBS code is owned by the Institut Français du Pétrole, the Université Paris Sud and the CNRS. It is developed in collaboration between those three owners and the CEA.
} 
Table 2. Relative differences for MCRSR and improved KLRR averaged over the isotherms $\left(T^{*}=2.5-5-\right.$ $20-100)$.

\begin{tabular}{c|cc|cc}
\hline & \multicolumn{2}{|c|}{$\delta Z(\%)$} & \multicolumn{2}{c}{$\delta U(\%)$} \\
\hline$\alpha$ & MCRSR & KLRR & MCRSR & KLRR \\
\hline 10.5 & 3.85 & 1.14 & 1.89 & 1.28 \\
11.5 & 3.00 & 0.78 & 2.13 & 1.38 \\
13.5 & 1.92 & 0.62 & 2.09 & 0.91 \\
15.5 & 1.66 & 0.79 & 2.98 & 1.26 \\
17.5 & 1.33 & 0.51 & 2.88 & 0.90 \\
\hline all & 2.35 & 0.77 & 2.39 & 1.15 \\
\hline
\end{tabular}

As previously mentioned, the improved KLRR method is more accurate than MCRSR. While KLRR calculations are very close to Monte Carlo data for $\alpha=17.5$ (at all temperatures), some discrepancies can be noticed for $\alpha=10.5$ at $T^{*}=2.5$. The KLRR isotherms $T^{*}=2.5$ for $\alpha=11.5-13.5-15.5$ are in fair agreement with Monte Carlo results. Relative differences averaged over the isotherms for the different values of $\alpha$ are reported in Table 2. The global relative difference is $0.96 \%$ for improved KLRR and $2.37 \%$ for MCRSR. This confirms that MCRSR is an accurate EOS and that improved KLRR method is notably much more accurate than MCRSR.

\section{Treatment of mixtures}

Considering the results of the literature, it has appeared that there is no analytical way to obtain a mixing rule for EXP-6 fluids [10]. As a consequence, we decided to fit the mixing rules on the thermodynamical properties of real mixtures computed by Monte Carlo simulations. With the GIBBS simulation code, we have computed the pressure and the energy at fixed molar volume and fixed temperature for different chemical compositions of binaries. Then the accuracy of the original VdW-1f mixing rule is evaluated in comparison with these reference data. Finally we propose a new mixing rule obtained by optimization of some coefficients on these reference data.

\subsection{Binary mixtures database}

The parameters of four fluids have been defined in order to sweep a large range of values of $\alpha, \epsilon$ and $\sigma$ [12] (hence they do not accurately reproduce the properties of the real fluids). The temperatures and pressures occuring in the Monte Carlo simulations correspond roughly to shocked states, that is temperatures of 2500 to $3500 \mathrm{~K}$ and pressures of 1 to $50 \mathrm{GPa}$. The cross parameters are obtained by the well-known Lorentz-Berthelot rules. We also recalculated the $H_{2}-H e$ isotherm $(T=1000 \mathrm{~K}$ and $V_{m}=9 \mathrm{~cm}^{3} \cdot \mathrm{mol}^{-1}$ ) as done by Ree [11].

As previously mentioned, comparing total pressure and total energy does not seem to be a good idea since the excess part of pressure/energy is small. Given a binary mixture of $A$ and $B$ at temperature $T$, molar volume $V_{m}$ and chemical composition $x=x_{A}=1-x_{B}$, one can define the ideal pressure $P_{\text {ideal }}$, the excess pressure $P_{\text {excess }}$ and the ideality discrepancy $\Delta P_{\text {excess }}$ as:

$$
\begin{gathered}
P_{\text {ideal }}(x)=x \cdot P_{A}+(1-x) \cdot P_{B} \\
P_{\text {excess }}(x)=P(x)-P_{\text {ideal }}(x) \\
\Delta P_{\text {excess }}(x)=\frac{P_{\text {excess }}(x)}{P_{\text {ideal }}(x)}
\end{gathered}
$$

where $P_{A}=P(x=1)$ and $P_{B}=P(x=0)$. The relevant quantities here are the excess pressure and the ideality discrepancy. 


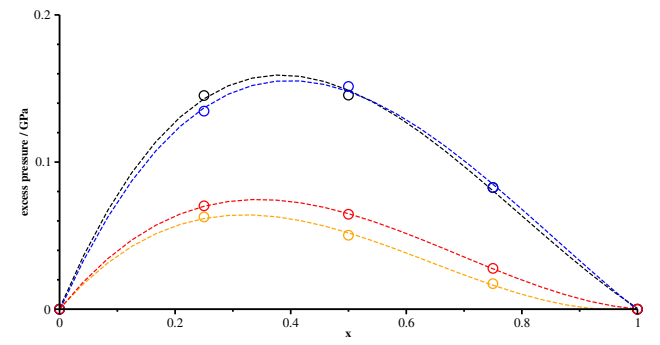

Fig. 4. Excess pressure for a binary mixture. Black symbols: Monte Carlo simulations of the real mixture. Red symbols: Monte Carlo simulations of the effective fluid. Orange symbols: improved KLRR calculations of the effective fluid. Blue symbols: improved KLRR calculations with the optimized mixing rule. Dashed lines are guide for the eyes.

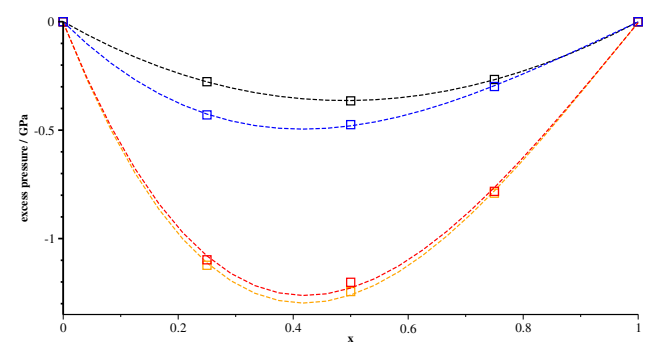

Fig. 5. Excess pressure for an other binary mixture. Black symbols: Monte Carlo simulations of the real mixture. Red symbols: Monte Carlo simulations of the effective fluid. Orange symbols: improved KLRR calculations of the effective fluid. Blue symbols: improved KLRR calculations with the optimized mixing rule. Dashed lines are guide for the eyes.

\subsection{Optimization procedure}

We suppose here that the analytical form proposed by Ree is valid. We only optimize the powers $(a, b, c, d, e, f)$ of the three rules: $\sigma_{x}^{a}=\sum_{i j} x_{i} x_{j} \sigma_{i j}^{a}, \epsilon_{x}^{c} \sigma_{x}^{b}=\sum_{i j} x_{i} x_{j} \epsilon_{i j}^{c} \sigma_{i j}^{b}$ and $\alpha_{x}^{f} \epsilon_{x}^{e} \sigma_{x}^{d}=\sum_{i j} x_{i} x_{j} \alpha_{i j}^{f} \epsilon_{i j}^{e} \sigma_{i j}^{d}$. The optimization procedure is as described in [4]. The pressures and energies of the effective fluids should be computed by Monte Carlo simulations, but this way requires an overwhelming computer time. To circumvent this difficulty, we computed the pressures and energies of the effective fluids with the improved KLRR theory. We checked in the case of VdW-1f mixing rules that excess properties computed by Monte Carlo simulations and by improved KLRR method are almost equal (Figs. 4 and 5). This results clearly shows that the VdW-1f rule is not very efficient for reproducing excess properties of binary mixtures. Moreover one can see that Monte Carlo simulation results and KLRR calculations of the effective fluids are consistent.

\subsection{Results}

Compared to the VdW-1f, the error function is decreased by a factor close to 4 and the powers obtained are $(3.151,3.087,1.517,3.547,0.249,1.087)$. The excess pressures computed with the optimized mixing rule are reported in blue in Figs. 4 and 5. Excess pressures computed with the optimized mixing rule are notably closer to Monte Carlo data than the ones computed with the VdW-1f rule. The same holds for reduced energies.

This new mixing rule has been optimized on thermodynamical properties of fluids at pressures and temperatures corresponding to shocked states. We do not intend to say that this rule can reproduce excess properties of fluids in standard states but we want to point out that the good old VdW-1f mixing rule, although largely used, may not be suited in all cases. A wider evaluation of the mixing rules should be initiated (analytical forms, powers,...). 


\section{Conclusion}

An evaluation of the MCRSR and improved KLRR perturbation methods has been realized on an extended Monte Carlo simulation database. It has been shown that the improved KLRR EOS is particularly accurate even at low temperatures. This method has then been implemented in the CARTE thermochemical code dedicated to the calculation of the thermodynamical properties of detonation products. In a second step, the largely used Van der Waals one fluid mixing rule, aimed at computing properties of effective fluids, has been carefully checked in comparison with Monte Carlo simulation data of binary mixtures. It appears that the original VdW-1f rule is not very accurate at reproducing excess properties of these binaries. In this paper, we have proposed an optimized mixing rule that may be a better choice in the case of detonation products mixtures.

\section{References}

1. M. Ross, J. Chem. Phys. 71, (1979) 1567

2. H.S. Kang and C.S. Lee and T. Ree and F.H. Lee, J. Chem. Phys. 82, (1985) 414

3. F. Charlet and M.-L. Turkel and J.-F. Danel and L. Kazandjian, J. Applied Phys., 84, (1998) 4227

4. N. Desbiens and V. Dubois, these proceedings

5. G. Zerah and J.-P. Hansen, J. Chem. Phys. 84, (1986) 2336

6. L. E. Fried and W. M. Howard, J. Chem. Phys. 109, (1998) 7338

7. S.B. Victorov and S.A. Gubin, In 13th International Detonation Symposium, (2006)

8. H.C. Longuet-Higgins, Proc. Roy. Soc. (London) A 205, (1951) 247

9. E.Z. Hamad and G.A. Mansoori, J. Chem. Phys. 87, (1987) 6046

10. S.F. Ragab and A.A. Helmy and T.L. Hassanein and M.A. El-Naggar, J. of Low Temperature Physics 111, (1998) 447

11. F.H. Ree, J. Chem. Phys. 78, (1983) 409

12. N. Desbiens and V. Dubois, to be published 